\title{
Non-Stationary Responses of Cables with Slowly Varying Length*
}

\author{
Stefan Kaczmarczyk ${ }^{\dagger}$ and \\ School of Mechanical Engineering, University of Natal, Durban 4041, Republic of South Africa \\ Wieslaw Ostachowicz
Institute of Fluid Flow Machinery, Polish Academy of Sciences, 14 Fiszera Street, 80-952 Gdansk, Poland
}

(Received 5 January 2000; accepted 12 April 2000)

\begin{abstract}
In this paper the longitudinal dynamics of hoisting cables with time-varying length is investigated. The overall response of a hoisting cable system to inertial load due to transport motion acceleration/deceleration and to external harmonic excitation is studied. A mathematical model of the system is formulated, and the response is simulated numerically. Due to the time-varying length of the cable, the natural frequencies of the system vary slowly, and a transient resonance occurs when one of the frequencies coincides with the frequency of the excitation at some critical time. Near the resonance region the resonant mode is dominant and a single-mode approximation of the system response in this region is formulated. A combined perturbation and numerical technique is applied to predict the response during passage through the main resonance. A numerical study is presented in which the dynamic responses of cables in a deep mine hoisting installation are examined.

${ }^{*}$ This article is an extended version of a paper presented by the first author at the Sixth International Congress on Sound and Vibration, Copenhagen, July 1999.

${ }^{\dagger}$ Member of the International Institute of Acoustics and Vibration (IIAV)
\end{abstract}

\section{INTRODUCTION}

Structural elements such as ropes and cables are among the oldest tools used by humanity in its quest for technological advancement. For example, a copper wire rope was found in the ruins of Nemeveh near Babylon which originate from about 700 B.C., ${ }^{1}$ and in Pompeii bronze ropes estimated to be 2400 years old have been excavated. ${ }^{2}$ These elements are known to have the ability to withstand relatively large axial loads in comparison to bending and torsional loads, and have played an indispensable role in towing operations, in supporting structures, in conducting signals and in systems designed to carry payloads in vertical and inclined transport installations. In this latter application cables are of timevarying length. However, the rate of change is small and the length may be assumed to vary slowly. Consequently, the dynamic characteristics of the system vary slowly during its operation, rendering the system non-stationary.

The responses of systems with non-stationary parameters and excitations are qualitatively different from the responses of stationary systems, especially in the neighbourhood of some critical values of the parameters, when transitions through resonance regions occur. The non-stationary resonance phenomena are often delayed, and frequently accompanied by beat phenomena. Hence, specialised treatment is required in order to analyse the responses of these systems. A number of studies have been carried out in this area. Kevorkian ${ }^{3}$ has considered the passage through resonance in a harmonically excited single-degree-of-freedom system with a slowly varying natural frequency. In this study the solution was constructed by matching two asymptotic expansions: the outer expansion away from resonance, and the inner expan- sion near resonance. Agrawal and Evan-Iwanowski, ${ }^{4}$ and Evan-Iwanowski ${ }^{5}$ have extended the asymptotic method developed by Mitropolskii ${ }^{6}$ for determining resonant responses of non-stationary, non-linear multi-degree-of-freedom systems. The theory and methodology to describe the behaviour of a system evolving slowly through internal resonance has been presented by Ablowitz, Funk and Newell, ${ }^{7}$ and also by Kevorkian. ${ }^{8}$

More recently Kevorkian, ${ }^{9}$ Bosley and Kevorkian ${ }^{10}$ and Bosley ${ }^{11}$ have proposed that in order to generate an approximate solution, the slowly varying oscillatory second-order system of $N$ equations can be transformed into a Hamiltonian standard form of $2 N$ first-order differential equations using action and angle variables together with the concept of adiabatic invariance. Later perturbation techniques, namely the method of averaging or the method of multiple scales, can be applied to determine the solution.

Alternatively, the perturbation techniques can be applied directly to the second-order model so that a first-order system can be obtained to compute the slowly varying amplitudes and phases for the first approximation of the response. Nayfeh and Asfar, ${ }^{12}$ and Neal and Nayfeh ${ }^{13}$ used this methodology to study single-degree-of-freedom systems with nonstationary parametric excitations. This technique was also implemented by Tran and Evan-Iwanowski ${ }^{14}$ to study the response of the Van der Pol oscillator with non-stationary external excitation, and by Cveticanin ${ }^{15}$ in the analysis of nonstationary oscillations of a textile machine rotor.

In this study the longitudinal response to an external excitation of a deep mine hoisting cable with slowly varying length and carrying concentrated inertial elements is investigated. The winding cycle in this hoisting installation consists of three main phases: the acceleration phase, the constant ve- 